\title{
An Investigation of the Limiting Behavior of Particle-like Solutions to the Einstein-Yang/Mills Equations and a New Black Hole Solution
}

\author{
J.A. Smoller, A.G. Wasserman \\ Department of Mathematics, University of Michigan, Ann Arbor, MI 48109-1003, USA
}

Received: 4 May 1994/in revised form: 2 May 1995

Commun. Math. Phys. 161, 365-389 (1994)

Theorems 4.1, Corollary 4.3, Theorem 4.4, Proposition 4.6 and Corollary 4.6 of the above paper are incorrect. The corrected version is summarized by:

Theorem. If $\left\{\Lambda_{n}(r)\right\}$ is a sequence of radially symmetric black hole solutions to the Einstein-Yang/Mills Equations (particle-like solutions if $\rho=0$ ) and if $\lim _{n \rightarrow \infty} \Omega\left(\Lambda_{n}\right)=\infty$, then for $r>1, \lim _{n \rightarrow \infty} \Lambda_{n}(r)=(0,0, \bar{A}(r), r)$, where $\bar{A}(r)=$ $1-\frac{2}{r}+\frac{1}{r^{2}}$ if $\rho \leqq 1$ and $\bar{A}(r)=1-\frac{\left(\rho+\rho^{-1}\right)}{r}+\frac{1}{r^{2}}$ if $\rho \geqq 1$. Moreover, the convergence is uniform on bounded $r$ intervals.

In other words, any sequence of black hole solutions with event horizon $\rho \geqq 0$ and increasing rotation numbers, must converge to an appropriate Riessner-Nordström solution for $r>1$. (If $\rho \leqq 1$ then these black hole solutions must converge to the critical Riessner-Nordström solution for $r>1$.)

The results in Sects. 2, 3, and 5 of the above paper are correct as stated, as are Lemmas 4.2 and 4.5. Section 6 is no longer relevant in view of the above theorem. The error is in the paragraph preceding Theorem 4.1 of the above paper; the authors assert that $p \neq(0,0)-$ in fact, $P=(0,0)$. P. Breitenlohner and D. Maison have also found this error and their proof of the above theorem in the case $\rho<1$ will appear shortly. 